\title{
Are Reducts and Typical Testors the Same?^
}

\author{
Manuel S. Lazo-Cortés ${ }^{1}$, José Fco. Martínez-Trinidad ${ }^{1}$, \\ Jesús Ariel Carrasco-Ochoa ${ }^{1}$, and Guillermo Sanchez-Diaz ${ }^{2}$ \\ 1 Instituto Nacional de Astrofísica, Óptica y Electrónica, México \\ 2 Universidad Autónoma de San Luis Potosí, México \\ $\{\mathrm{mlazo}$, fmartine, ariel\}@inaoep.mx, guillermo.sanchez@uaslp.mx
}

\begin{abstract}
This paper deals with the relation between rough set reducts and typical testors from the logical combinatorial approach to pattern recognition. The main objective is to clarify once and for all that although in many cases the two concepts coincide, being rigorous they are not the same. Definitions, comments and observations are formally introduced and supported by illustrative examples. Furthermore, some theorems expressing theoretical relations between reducts and typical testors are enunciated and proved.
\end{abstract}

\section{Introduction}

This paper deals with the relation between rough set reducts 8] and typical testors [13. from the so-called logical combinatorial approach to pattern recognition [11. The assumption that both concepts are closely related (to the point that some authors equate them) is based on their common properties. In both approaches, decision tables are used for data representation, it means that objects of study are perceived by means of information represented by attributes. Both, reducts and typical testors (some authors use test instead of testor), represent strong differentiating power, they are attribute subsets jointly sufficient and individually necessary to discern among object descriptions.

As an important antecedent and a motivation, we can mention the work of M. Ju. Moshkov (see for example 44), who has deeply studied decision trees and relations among testors, decision rules and decision trees. In this publication, several assertions like "A reduct ... is a test ... for which each proper subset is not a test" ( page 6) or "we use the term test instead of the term super reduct" (page 3) can be read.

The contribution of this study is to make clear, that although there is a close relationship between couples testor-super reduct and typical testor-reduct, actually they have different scopes and in some cases they differ.

This document is organized as follows. Section 2 provides the formal background for the study. Section 3 contains theorems expressing relations between reducts and typical testors, including a case study. Our remarks are summarized in Section 5 .

\footnotetext{
* This work was partly supported by the National Council of Science and Technology of Mexico (CONACyT) through the project grant CB2008-106366.
} 


\section{Basic Concepts}

\section{$2.1 \quad$ Reducts}

In many data analysis applications, information and knowledge are stored and represented as a decision table which provides a convenient way to describe a finite set of objects within a universe through a finite set of attributes 8 .

Definition 1. A decision table is a pair $\mathcal{S}_{d}=\left(U, A_{t}=A_{t}^{*} \cup\{d\}\right)$, where $U$ is a set of objects, $A_{t}^{*}$ is a set of conditional attributes and $d$ is a decision attribute $(\{d\}=D)$. Each $a \in A_{t}$ corresponds to the function $I_{a}: U \rightarrow V_{a}$ called evaluation function, where $V_{a}$ is called the value set of a

Definition 2. Given a subset of conditional attributes $A \subseteq A_{t}^{*}$, the indiscernibility relation is defined as $I N D(A \mid D)=\left\{(u, v) \in U \times U: \forall a \in A,\left[I_{a}(u)=\right.\right.$ $\left.\left.I_{a}(v)\right] \vee\left[I_{d}(u)=I_{d}(v)\right]\right\}$.

In practice, it is common that decision tables contain descriptions of a finite sample $U$ of objects from a larger (possibly infinite) universe $\mathcal{U}$, where values of descriptive attributes are always known for all objects from $\mathcal{U}$, but the decision attribute is in general a hidden function except for those objects from the sample $U$. The main problem of learning theory is to generalize the decision function (defined on the sample $U$ ) to the whole universe $\mathcal{U}$.

Based on the indiscernibility relation, a reduct $[9]$ is defined as follows.

Definition 3. Given a decision table $\mathcal{S}_{d}$, an attribute set $R \subseteq A_{t}^{*}$ is called a reduct, if $R$ satisfies the following two conditions:

(i) $I N D(R \mid D)=I N D\left(A_{t}^{*} \mid D\right)$ (if $R$ satisfies (i) it is called a super reduct);

(ii) For any $a \in R, I N D((R-\{a\}) \mid D) \neq I N D\left(A_{t}^{*} \mid D\right)$.

Another widely used definition of reduct requires that a region of the universe be preserved, this region is called the positive region. Next, we will introduce it.

Definition 4. Let $A \subseteq A_{t}$, and let $I N D(A)=\left\{(u, v) \in U \times U: \forall a \in A, I_{a}(u)=\right.$ $\left.I_{a}(v)\right\} ;[u]_{A}$ denotes the class in $U / I N D(A)$ containing $u$. We define the $A$ lower approximation of a set $X \subseteq U$ as $\underline{A}(X)=\left\{u \in U:[u]_{A} \subseteq X\right\}$.

Definition 5. Given a decision table $\mathcal{S}_{d}$ and a subset of attributes $A \subseteq A_{t}^{*}$. The $A$-positive region with respect to $d$ is defined as

$$
\operatorname{POS}_{\{d\}}(A)=\cup_{X \in U / I N D(\{d\}) \underline{A}(X) .}
$$

We say that a decision table $\mathcal{S}_{d}$ is consistent if $\operatorname{POS}_{\{d\}}\left(A_{t}^{*}\right)=U$. Otherwise, we call it inconsistent. Usually, the following definition of reduct is used [12].

Definition 6. Given a decision table $\mathcal{S}_{d}$, an attribute subset $R \subseteq A_{t}^{*}$ is called a relative reduct, if $R$ satisfies the two conditions: 
(i) $P O S_{\{d\}}(R)=P O S_{\{d\}}\left(A_{t}^{*}\right)$; ( $R$ is called a relative super reduct)

(ii) $\operatorname{POS}_{\{d\}}(R-\{a\}) \neq \operatorname{POS}_{\{d\}}\left(A_{t}^{*}\right)$ for any $a \in R$.

An important point for answering the question are reducts and typical testors the same? is the study of the relationship between these two ways of defining a reduct for a decision table, and the understanding that the two definitions are equivalent when the decision table is consistent but not in the general case, as it is shown in the following example.

Example 1. Let us consider the decision table in Table 1 (a) where $U=\left\{u_{1}\right.$, $\left.u_{2}, u_{3}, u_{4}, u_{5}, u_{6}, u_{7}\right\}, A_{t}^{*}=\left\{a_{1}, a_{2}, a_{3}, a_{4}\right\}$ and $D=\{d\}$. We have that $I N D\left(A_{t}^{*} \mid D\right)=\left\{\left(u_{1}, u_{1}\right),\left(u_{2}, u_{2}\right),\left(u_{3}, u_{3}\right),\left(u_{4}, u_{4}\right),\left(u_{5}, u_{5}\right),\left(u_{6}, u_{6}\right),\left(u_{7}, u_{7}\right)\right.$, $\left\langle u_{1}, u_{2}\right\rangle,\left\langle u_{1}, u_{3}\right\rangle,\left\langle u_{1}, u_{4}\right\rangle,\left\langle u_{2}, u_{3}\right\rangle,\left\langle u_{2}, u_{4}\right\rangle,\left\langle u_{3}, u_{4}\right\rangle,\left\langle u_{5}, u_{6}\right\rangle,\left\langle u_{5}, u_{7}\right\rangle,\left\langle u_{6}, u_{7}\right\rangle$, $\left.\left\langle u_{2}, u_{6}\right\rangle,\left\langle u_{3}, u_{6}\right\rangle,\left\langle u_{4}, u_{5}\right\rangle\right\}$, where $\left\langle u_{i}, u_{j}\right\rangle$ denotes the two elements $\left(u_{i}, u_{j}\right)$ and $\left(u_{j}, u_{i}\right)$.

Consider the attribute subset $R=\left\{a_{1}, a_{2}, a_{3}\right\}$, then $I N D(R)=\left\{\left(u_{1}, u_{1}\right)\right.$, $\left(u_{2}, u_{2}\right),\left(u_{3}, u_{3}\right),\left(u_{4}, u_{4}\right),\left(u_{5}, u_{5}\right),\left(u_{6}, u_{6}\right),\left(u_{7}, u_{7}\right),\left\langle u_{2}, u_{3}\right\rangle,\left\langle u_{2}, u_{6}\right\rangle,\left\langle u_{3}, u_{6}\right\rangle$, $\left.\left\langle u_{4}, u_{5}\right\rangle\right\}=I N D\left(A_{t}^{*}\right)$, therefore $I N D(R \mid D)=I N D\left(A_{t}^{*} \mid D\right)$. Following Definition 3 is a super reduct for this decision table. Let analyze if $R$ is a reduct, and for this purpose let consider the subsets $R_{i}=R-\left\{a_{i}\right\} ; i=1,2,3$, for which we have that $I N D\left(R_{1} \mid D\right)=I N D\left(A_{t}^{*} \mid D\right) \cup\left\{\left\langle u_{2}, u_{5}\right\rangle,\left\langle u_{3}, u_{5}\right\rangle,\left\langle u_{4}, u_{6}\right\rangle\right\} ; I N D\left(R_{2} \mid D\right)$ $=I N D\left(A_{t}^{*} \mid D\right) \cup\left\{\left\langle u_{1}, u_{7}\right\rangle\right\}$ and $I N D\left(R_{3} \mid D\right)=I N D\left(A_{t}^{*} \mid D\right) \cup\left\{\left\langle u_{1}, u_{6}\right\rangle\right\}$.

According to condition (ii) in Definition 3 , we can say that $R$ is a reduct for this decision table.

Now, let us consider the Definition 6] then $U / I N D(\{d\})=\left\{\left\{u_{1}, u_{2}, u_{3}, u_{4}\right\}\right.$, $\left.\left\{u_{5}, u_{6}, u_{7}\right\}\right\} ; U / I N D\left(A_{t}^{*}\right)=\left\{\left\{u_{1}\right\},\left\{u_{2}, u_{3}, u_{6}\right\},\left\{u_{4}, u_{5}\right\},\left\{u_{7}\right\}\right\}$ and $\underline{A}_{t}^{*}\left(\left\{u_{1}\right.\right.$, $\left.\left.u_{2}, u_{3}, u_{4}\right\}\right)=\left\{u_{1}\right\}=\underline{R}\left(\left\{u_{1}, u_{2}, u_{3}, u_{4}\right\}\right) ; \underline{A}_{t}^{*}\left(\left\{u_{5}, u_{6}, u_{7}\right\}\right)=\left\{u_{7}\right\}=\underline{R}\left(\left\{u_{5}\right.\right.$, $\left.u_{6}, u_{7}\right\}$ ), and therefore $\operatorname{POS}_{\{d\}}\left(A_{t}^{*}\right)=\left\{u_{1}, u_{7}\right\}=P O S_{\{d\}}(R)$; so $R$ satisfies condition (i) of Definition 6 and we can say that $R$ is a relative super reduct.

Let analyze condition (ii); $U / I N D\left(R_{1}\right)=\left\{\left\{u_{1}\right\},\left\{u_{2}, u_{3}, u_{4}, u_{5}, u_{6}\right\},\left\{u_{7}\right\}\right\}$; $U / I N D\left(R_{2}\right)=\left\{\left\{u_{1}, u_{7}\right\},\left\{u_{2}, u_{3}, u_{6}\right\},\left\{u_{4}, u_{5}\right\}\right\}$ and $U / I N D\left(R_{3}\right)=\left\{\left\{u_{1}, u_{2}\right.\right.$, $\left.\left.u_{3}, u_{6}\right\},\left\{u_{4}, u_{5}\right\},\left\{u_{7}\right\}\right\}$; then $\underline{R}_{1}\left(\left\{u_{1}, u_{2}, u_{3}, u_{4}\right\}\right)=\left\{u_{1}\right\}, \underline{R}_{2}\left(\left\{u_{1}, u_{2}, u_{3}, u_{4}\right\}\right)$ $=\emptyset$ and $\underline{R}_{3}\left(\left\{u_{1}, u_{2}, u_{3}, u_{4}\right\}\right)=\emptyset$ and $\underline{R}_{1}\left(\left\{u_{5}, u_{6}, u_{7}\right\}\right)=\left\{u_{7}\right\}, \underline{R}_{2}\left(\left\{u_{5}, u_{6}, u_{7}\right\}\right)$

Table 1. Two examples of decision tables

(a)

\begin{tabular}{cccccc}
\hline$U$ & $a_{1}$ & $a_{2}$ & $a_{3}$ & $a_{4}$ & $d$ \\
\hline$u_{1}$ & 1 & 0 & 1 & 0 & 0 \\
$u_{2}$ & 1 & 0 & 0 & 1 & 0 \\
$u_{3}$ & 1 & 0 & 0 & 1 & 0 \\
$u_{4}$ & 0 & 0 & 0 & 1 & 0 \\
$u_{5}$ & 0 & 0 & 0 & 1 & 1 \\
$u_{6}$ & 1 & 0 & 0 & 1 & 1 \\
$u_{7}$ & 1 & 1 & 1 & 1 & 1
\end{tabular}

(b)

\begin{tabular}{ccccc}
\hline$U$ & $a_{1}$ & $a_{2}$ & $a_{3}$ & $d$ \\
\hline$u_{1}$ & 1 & & 1 & hor 0 \\
$u_{2}$ & 0 & 0 & hor 0 \\
$u_{3}$ & 0 & 1 & ver 0 \\
$u_{4}$ & 1 & 1 & neu 1 \\
$u_{5}$ & 0 & 0 & hor 1 \\
$u_{6}$ & 1 & 1 & ver 0
\end{tabular}


$=\emptyset$ and $\underline{R}_{3}\left(\left\{u_{5}, u_{6}, u_{7}\right\}\right)=\left\{u_{7}\right\}$; thence $P O S_{\{d\}}\left(R-\left\{a_{1}\right\}\right)=P O S_{\{d\}}\left(A_{t}^{*}\right)$. It means that $R$ does not fulfill condition (ii) of Definition 6 . We can conclude that $R$ is a reduct of the decision table in Table 1 (a) according to Definition 3 but it is not a reduct if we use Definition 6 .

It should be emphasized that although in some publications the equivalence between both definitions of reduct here included (3) and 6) is handled lightly; in 12 it is accurately established (Proposition 5.6, page 351) that if $I N D\left(A_{t}^{*}\right)=$ $I N D(\{d\})$ the equivalence holds. Meanwhile, Bazan and Szczuka 2] also remark that in the presence of an inconsistent decision table the notion of generalized decision, which is defined below, has to be used.

There are other definitions of reducts. Miao et al. [7] for example, define a reduct as a minimum set of attributes that preserve certain property (e.g. indiscernibility relation, generalized decision, positive region, etc.).

Definition 7. Let $\mathcal{S}_{d}$ be a decision table, the generalized decision function $\partial$ is defined as $\partial(u)=\left\{k \in V_{d}: \exists v \in U\left[(u, v) \in I N D\left(A_{t}^{*}\right) \wedge I_{d}(v)=k\right]\right\}$.

Now, we can say that $\mathcal{S}_{d}$ is consistent if $|\partial(u)|=1$ for any $u \in U$.

An important consequence of the last definition is that one can transform an arbitrary inconsistent decision table $\mathcal{S}_{d}=\left(U, A_{t}=A_{t}^{*} \cup\{d\}\right)$ into a consistent decision table $\mathcal{S}_{\partial}=\left(U, A_{t}^{*} \cup\{\partial\}\right)$.

We will differentiate between the reducts defined by Definition 3 (we will call them discerning decision reducts and we will denote the set of all these reducts by $R E D_{\text {ind }}\left(\mathcal{S}_{d}\right)$ ) and those defined by Definition [6 (which we will call positive region decision reducts, and we will denote the set of all of them by $R E D_{\text {pos }}\left(\mathcal{S}_{d}\right)$ ), analogously we will denote as $D S_{\text {ind }}\left(\mathcal{S}_{d}\right)$ and $D S_{\text {pos }}\left(S_{d}\right)$ the set of super reducts determined by the definitions 3 and 6 respectively. Sometimes, to point out that the generalized decision is being used, we will use the notation $\mathcal{S}_{\partial}$ instead of $\mathcal{S}_{d}$.

Being $\mathcal{S}_{\partial}$ a consistent table, we can derive the following fact:

Lemma 1. Let $\mathcal{S}_{d}$ a decision table, then

$$
R E D_{\text {pos }}\left(\mathcal{S}_{d}\right)=R E D_{\text {ind }}\left(\mathcal{S}_{\partial}\right)=R E D_{\text {pos }}\left(\mathcal{S}_{\partial}\right) .
$$

The below theorem asserts an interesting result.

Theorem 1. Let $\mathcal{S}_{d}$ a decision table, then $D S_{\text {ind }}\left(\mathcal{S}_{d}\right) \subseteq D S_{\text {pos }}\left(\mathcal{S}_{\partial}\right)$. If $\mathcal{S}_{d}$ is consistent, the equality holds.

It is important to highlight that sometimes the equality holds even if we do not have a consistent table, as it can be seen from Example 2 .

Example 2. Consider the decision table $\mathcal{S}_{d}$ in Table 1 (b) being $U=\left\{u_{1}, u_{2}, u_{3}\right.$, $\left.u_{4}, u_{5}, u_{6}\right\}, A_{t}^{*}=\left\{a_{1}, a_{2}, a_{3}\right\}$ and $D=\{d\}$. Notice that Table 1 (b) is inconsistent since $I_{\left\{a_{1}, a_{2}, a_{3}\right\}}\left(u_{2}\right)=I_{\left\{a_{1}, a_{2}, a_{3}\right\}}\left(u_{5}\right)$ but $I_{d}\left(u_{2}\right) \neq I_{d}\left(u_{5}\right)$. The reader can verify that despite being an inconsistent table, $R E D_{\text {pos }}\left(\mathcal{S}_{d}\right)=\left\{\left\{a_{2}, a_{3}\right\},\left\{a_{1}, a_{3}\right\}\right\}$ $=R E D_{\text {ind }}\left(\mathcal{S}_{d}\right)$. 


\subsection{Testors}

The concept of testor was created by S. V. Yablonskii [13 as a tool for solving problems of control and diagnosis of faults in contact networks. From the early works 313 , a research line that is primarily concerned with this kind of problems was derived. In publications related to this area the original term test is used instead of testor and the minimal ones (typical testors) are called dead-end tests. The concept of testor (and typical testor) has had numerous generalizations and adaptations to different environments [6]. In this paper, we focus on the classical concept [5].

Let $U$ and $A_{t}$ be a set of objects and a set of descriptive attributes respectively. $V_{a}$ denotes the domain of the attribute $a$ and $I_{a}(u)$ the value of the attribute $a \in A_{t}$ for the object $u \in U$. Let $M=(M[i, j])_{m \times n}$ be the matrix representation of the information. Thus, the element $M[i, j]=I_{a_{j}}\left(u_{i}\right)$ represents the value of the attribute $a_{j}$ for the object $u_{i}$.

Let us consider a supervised classification problem and let us assume that we have information represented as a decision table $\mathcal{S}_{d}=\left(U, A_{t}^{*} \cup\{d\}\right)$ being $d$ a decision attribute $\left(d \notin A_{t}^{*}\right)$. $\mathcal{S}_{d}$ is known as a training sample and its matrix representation as a training matrix.

Let $T \subseteq A_{t}^{*}$ and let $I_{T}(u)$ denote the partial description of $u$ considering only attributes belonging to $T$. In this framework, we will define a testor as:

Definition 8. $T \subseteq A_{t}^{*}$ is a testor with respect to a training matrix if $\forall u, v \in U$ : $\left[I_{T}(u)=I_{T}(v)\right] \Rightarrow\left[I_{d}(u)=I_{d}(v)\right]$. If $T$ is a testor such that none of its proper subsets is a testor, then $T$ is a typical (irreducible) testor.

This definition means that attributes belonging to a testor are jointly sufficient to discern between any pair of objects belonging to different classes, that is exactly the same as a discerning decision super reduct. If a testor is typical, each attribute is individually necessary in the same way as in a discerning decision reduct; then we can enunciate the following lemma.

Lemma 2. Let $\mathcal{S}_{d}=\left(U, A_{t}^{*} \cup\{d\}\right)$ be a decision table and let $\mathcal{M}$ be its associated training matrix. Let $R \subseteq A_{t}^{*}$, then

(i) $R$ is a discerning decision super reduct for $\mathcal{S}_{d}$ iff $R$ is a testor for $\mathcal{M}$.

(ii) $R$ is a discerning decision reduct for $\mathcal{S}_{d}$ iff $R$ is a typical testor for $\mathcal{M}$.

Although initially the concept of testor in supervised classification problems was associated with disjoint classes, in [10] non-disjoint classes are considered into the testor definition through the confusion concept.

Definition 9. Let $\mathcal{M}$ be a training matrix and $a_{j}$ a descriptive attribute, the number of object pairs belonging to different classes that are indiscernible by the attribute $a_{j}$ is called the confusion induced by $a_{j}$ in $\mathcal{M}$ and we denote it by $\mathcal{C}\left(\left\{a_{j}\right\}\right)$. Similarly we can define the confusion induced by $A \subseteq A_{t}^{*}$ by $\mathcal{C}(A)=\left|\cap_{a_{j} \in A} \tilde{\mathcal{C}}\left(\left\{a_{j}\right\}\right)\right|$, being $\tilde{\mathcal{C}}\left(\left\{a_{j}\right\}\right)=\left\{(u, v) \in \mathcal{U} \times \mathcal{U}:\left[I_{a_{j}}(u)=I_{a_{j}}(v)\right] \wedge\right.$ $\left.\left[I_{d}(u) \neq I_{d}(v)\right]\right\}$. 
From this definition the following lemma is immediate.

Lemma 3. Let $T \subseteq A_{t}^{*}$. $T$ is a testor iff $\mathcal{C}(T)=0 . T$ is typical if for all $T^{\prime} \subset T, \mathcal{C}\left(T^{\prime}\right) \neq 0$.

Example 3. For matrix in Table 1 (b) we have that $\mathcal{C}\left(a_{1}\right)=\mathcal{C}\left(a_{2}\right)=8$ and $\mathcal{C}\left(a_{3}\right)=$ 4. Considering for example $T_{1}=\left\{a_{1}, a_{2}\right\}$, we have that $\mathcal{C}\left(T_{1}\right)=4$. Notice that $\tilde{\mathcal{C}}\left(\left\{a_{1}\right\}\right) \cap \tilde{\mathcal{C}}\left(\left\{a_{2}\right\}\right)=\left\{\left\langle u_{1}, u_{4}\right\rangle,\left\langle u_{2}, u_{5}\right\rangle\right\}$.

The definition of confusion was used to broaden the scope of application of the concept of testor to problems with overlapping classes. Although in this kind of problems the term irreducible combination of attributes is more commonly found, the term testor is also used and it is the one we use in this work for the purpose of homogeneity. Thus, the following definition is more general.

Definition 10. Let $T \subseteq A_{t}^{*} . T$ is a global testor if $\mathcal{C}(T)=\mathcal{C}\left(A_{t}^{*}\right)$. $T$ is typical if none of its proper subsets is a global testor, i.e. $\mathcal{C}\left(T^{\prime}\right) \neq \mathcal{C}\left(A_{t}^{*}\right)$ for all $T^{\prime} \subset T$.

Definition 8 becomes particular case of this one. We will denote by $T T(\mathcal{M})$ the set of all typical global testors of the training matrix $\mathcal{M} . T(\mathcal{M})$ will denote the set of all global testors of $\mathcal{M}$.

\section{Relations}

Finally, we present the theoretical relations between reducts and typical testors. Due to space constraints proofs are not included.

Theorem 2. Let $\mathcal{S}_{d}$ be a consistent decision table and $\mathcal{M}$ its associated training matrix; then $R E D_{\text {ind }}\left(\mathcal{S}_{d}\right)=R E D_{\text {pos }}\left(\mathcal{S}_{\partial}\right)=T T(\mathcal{M})$.

Theorem 3. Let $\mathcal{S}_{d}=\left(U, A_{t}^{*} \cup\{d\}\right)$ be a (possibly inconsistent) decision table and $\mathcal{M}$ its associated training matrix. Let $\mathcal{S}_{\partial}=\left(U, A_{t}^{*} \cup\{\partial\}\right)$ the consistent table obtained from $\mathcal{S}_{d}$ considering generalized decision and $\mathcal{M}_{\partial}$ the corresponding training matrix. Then $R E D_{\text {ind }}\left(\mathcal{S}_{d}\right)=T T(\mathcal{M})$ and $R E D_{\text {pos }}\left(\mathcal{S}_{d}\right)=R E D_{\text {pos }}\left(\mathcal{S}_{\partial}\right)$ $=T T\left(\mathcal{M}_{\partial}\right)$.

Let $\mathcal{S}_{d}=\left(U, A_{t}^{*} \cup\{d\}\right)$ be a (possibly inconsistent) decision table, and let us introduce the decision attribute $\Delta$ defined by

$$
\Delta(u)=\left\{\begin{array}{l}
{[u]_{\partial} \text { if }[u]_{\partial} \subseteq[u]_{\{d\}}} \\
{[u]_{A_{t}^{*}} \text { otherwise }}
\end{array}\right.
$$

Lemma 4. Let $\mathcal{S}_{d}=\left(U, A_{t}^{*} \cup\{d\}\right)$ be a decision table and let $\mathcal{S}_{\Delta}=\left(U, A_{t}^{*} \cup\{\Delta\}\right)$ being $\Delta$ defined as in (1), then $\mathcal{S}_{\Delta}$ is consistent.

From these results we have the following corollary which establishes a relation between the different partitions of $U$. 
Table 2. Typical testors and positive region decision reducts for the Spect dataset

\begin{tabular}{|c|c|c|c|c|c|}
\hline Data set & $\begin{array}{c}\text { Descriptive attributes C } \\
\text { (A) }\end{array}$ & $\begin{array}{l}\text { Classes } \\
\text { (B) }\end{array}$ & $\begin{array}{l}\text { Objects } \\
\text { (C) }\end{array}$ & $\begin{array}{l}\mid T T\left(\mathcal{M}_{*}\right) \\
\quad(\mathrm{D})\end{array}$ & $\begin{array}{c}|| R E D_{\text {pos }}\left(S_{*}\right) \mid \\
(\mathrm{E})\end{array}$ \\
\hline Spect $=\left(\mathcal{S}_{d}\right)$ & 22 & 2 & 80 & 26 & 70 \\
\hline Spect $3 c=\mathcal{S}_{\partial}$ & 22 & 3 & 80 & 70 & 70 \\
\hline Spect6c $=\mathcal{S}_{\Delta}$ & 22 & 6 & 80 & 26 & 26 \\
\hline
\end{tabular}

Corollary 1. With the same hypothesis as Lemma 4: $U / I N D\left(A_{t}^{*}\right)$ is a finer partition of $U$ than $U / I N D(\{\Delta\})$ while $U / I N D(\{\Delta\})$ is finer than $U / I N D(\{\partial\})$. It means that for every $u \in U,[u]_{A_{t}^{*}} \subseteq[u]_{\Delta} \subseteq[u]_{\partial}$. If $\mathcal{S}_{d}$ is consistent then $[u]_{A_{t}^{*}} \subseteq[u]_{\Delta}=[u]_{\partial}=[u]_{\{d\}}$.

It is easy to realize that:

Theorem 4. Let $\mathcal{S}_{d}=\left(U, A_{t}^{*} \cup\{d\}\right)$ be a decision table and let $\mathcal{S}_{\Delta}=\left(U, A_{t}^{*} \cup\right.$ $\{\Delta\})$ built as above. Let $\mathcal{M}$ and $\mathcal{M}_{\Delta}$ the respective associated training matrices, then $T T(\mathcal{M})=R E D_{\text {pos }}\left(\mathcal{S}_{\Delta}\right)$.

The theorems enunciated in this section confirm the non-equivalence between the concepts of reduct and typical testor. Even more, they give us the possibility of using algorithms to compute reducts in contexts where algorithms to compute testors are used and vice versa. For example, Theorem 4 allows computing discerning decision reducts (Def. 3) for an inconsistent table by using a software like RSES 2] designed for computing positive region decision reducts (Def. 6).

\subsection{Case Study}

Here, we illustrate the non-equivalence between typical testors and reducts using Spect dataset taken from the UCI Machine Learning Repository [1]. The Spect data set consists of two non-disjoint classes, each class containing 40 objects and 22 descriptive attributes, but there are several coincidences.

From the Spect dataset, we created two modified datasets: Spect3c and Spect6c. Spect3c results from creating a new class containing the intersection between the two original classes and Spect6c results from separating each repeated description as a new class. Notice that Spect3c is the table resulting from applying generalized decision; in Table 2 we denote it by $\mathcal{S}_{\partial}$, meanwhile Spect6c corresponds to $\mathcal{S}_{\Delta}$.

Table 2 presents the amount of typical testors (column D) and positive region decision reducts (column E) from the original Spect dataset and for the other two datasets before described. Although the table does not explicitly contain the attribute sets but only quantities, we can see the equalities $|T T(\mathcal{M})|=$ $\left|R E D_{\text {pos }}\left(S_{\Delta}\right)\right|$ and $\left|T T\left(\mathcal{M}_{\partial}\right)\right|=\left|R E D_{\text {ind }}\left(S_{d}\right)\right|$. For second and third rows the tables in the first column are consistent, for this reason in these rows the values in columns (D) and (E) are equal, i.e $\left|T T\left(\mathcal{M}_{*}\right)\right|=\left|R E D_{\text {pos }}\left(S_{*}\right)\right|$ with * taking value in $\{\partial, \Delta\}$. 


\section{Conclusions}

This paper studies the relation between reducts (from rough set theory) and typical testors (from logical combinatorial pattern recognition) and demonstrates that although in many cases (specially when classes are disjoint) the two concepts coincide, they are not exactly the same. The knowledge of the true relations between these concepts allows a proper use in one area of the results obtained in the other. This study can be a starting point for further considerations and will probably lead to interesting practical applications in which both research communities can benefit from each other. Although we focus on the relation between the classical concepts of testor and reduct, we believe that our study can be expanded to include other types of testors and reducts.

\section{References}

1. Bache, K., Lichman, M.: UCI Machine Learning Repository. University of California, School of Information and Computer Science, Irvine, CA (2013), http://archive.ics.uci.edu/ml

2. Bazan, J.G., Szczuka, M.S.: The Rough Set Exploration System. In: Peters, J.F., Skowron, A. (eds.) Transactions on Rough Sets III. LNCS, vol. 3400, pp. 37-56. Springer, Heidelberg (2005)

3. Cheguis, I.A., Yablonskii, S.V.: Logical methods of control of work of electric schemes. Trudy Mat. Inst. Steklov. 51, 270-360 (1958) (in Russian)

4. Chikalov, I., Lozin, V., Lozina, I., Moshkov, M., Nguyen, H.S., Skowron, A., Zielosko, B.: Three approaches to Data Analysis, Intelligent Systems Reference Library, vol. 41. Springer, Heidelberg (2013)

5. Dmitriev, A.N., Zhuravlev, Y.I., Krendelev, F.P.: On mathematical principles for objects and phenomena classification. Diskretny Analiz 7, 3-11 (1966) (in Russian)

6. Lazo-Cortes, M., Ruiz-Shulcloper, J., Alba-Cabrera, E.: An overview of the evolution of the concept of testor. Pattern Recognition 34(4), 753-762 (2001)

7. Miao, D.Q., Zhao, Y., Yao, Y.Y., Li, H.X., Xu, F.F.: Relative reducts in consistent and inconsistent decision tables of the Pawlak rough set model. Information Sciences 179(24), 4140-4150 (2009)

8. Pawlak, Z.: Rough sets. International Journal of Computer Information and Science 11, 341-356 (1982)

9. Pawlak, Z.: Rough Sets: Theoretical Aspects of Reasoning About Data. Kluwer Academic Publishers, Dordrecht (1991)

10. Ruiz-Shulcloper, J.: Some questions about pattern recognition problems with nondisjoint classes. Editorial Academia, Havana (1995) (in Spanish)

11. Ruiz-Shulcloper, J., Abidi, M.A.: Logical Combinatorial Pattern Recognition: A Review. In: Pandalai, S.G. (ed.) Recent Research Developments in Pattern Recognition, pp. 133-176. Transwold Research Network, Kerala (2002)

12. Skowron, A., Rauszer, C.: The discernibility matrices and functions in information systems. In: Słowiński, R. (ed.) Intelligent Decision Support, Handbook of Applications and Advances of the Rough Sets Theory, System Theory, Knowledge Engineering and Problem Solving, vol. 11, pp. 331-362. Kluwer Academic Publishers, Dordrecht (1992)

13. Yablonskii, S.V., Cheguis, I.A.: On tests for electric circuits. Uspekhi Mat. Nauk 10(4), 182-184 (1955) (in Russian) 